\title{
RAPID EVAPORATIVE IONIZATION MASS SPECTROMETRY FOR HIGH THROUGHPUT SCREENING IN FOOD ANALYSIS THE CASE OF BOAR TAINT
}

Kaat Verplanken*1, Sara Stead ${ }^{2}$, Renata Jandova ${ }^{2}$, Christof Van Poucke ${ }^{3}$, Jan Claereboudt ${ }^{4}$, Julie Vanden Bussche ${ }^{1}$, Sarah De Saeger ${ }^{5}$, Zoltan Takats ${ }^{6}$, Jella Wauters ${ }^{1}$ and Lynn Vanhaecke $^{1}$

Ghent University, Faculty of Veterinary Medicine, Department of Veterinary Public Health and Food Safety, Laboratory of Chemical Analysis, Salisburylaan 133, B-9820 Merelbeke, Belgium 2 Waters Corporation, Altrincham Road, UK-SK9 4AX Wilmslow, United Kingdkom

${ }^{3}$ Institute of Agricultural and Fischeries Research (ILVO), Technology and Food Sciences Unit, Brusselsesteenweg 370, B-9090 Melle, Belgium

4 Waters Corporation, Brusselsesteenweg 500, B

${ }^{4}$ Waters Corporation, Brusselsesteenweg 500, B- 1731 Zellik, Belgium
Department of Bioanalysis, Ghent University, Ottergemsesteenweg 460, Ghent, B-9000, Belgium

${ }^{6}$ Faculty of Medicine, Department of Surgery and Cancer, Imperial College London, London, SW7 2AZ, UK

*E-mail: Kaat.Verplanken@UGent.be Website: http://mww.vwv.ugent.be negative consumer reactions, and consequently leads to severe economic losses in pig husbandry. For this reason, it is crucial to timely detect boar taint containing carcasses at the slaughter line. Since currently, accurate and fast analysis methods at the slaughter line are lacking, rapid evaporative

ionization mass spectrometry (REIMS) was evaluated for the latter purpose.

\section{MATERIALS \& METHODS}

\section{Samples}

Sow and boar neck fat samples were collected at the slaughter line ( $n=150$ ). The presence or absence of boar taint in the samples was confirmed by sensory evaluation and UHPLC-HR-Orbitrap-MS analysis( ${ }^{(1)}$. Samples containing levels of indole (IND), skatole (SK) and androstenone (AEON) above and below the odour thresholds (IND: $100 \mu \mathrm{g} \mathrm{kg}{ }^{-1}, \mathrm{SK}: 200 \mu \mathrm{g} \mathrm{kg}{ }^{-1}, \mathrm{AEON}: 500 \mu \mathrm{g} \mathrm{kg}{ }^{-1}$ ) were considered as positive and negative for boar taint, respectively.

(1) Bekaert, K.M.; Vanden Bussche, J.; Francois,

\section{Instrumentation}

Sampling was carried out for 3 to 5 seconds with an iKnife hand-held sampling device (Waters, Wilmslow, UK), which was directly coupled to a Xevo G2-XS Q-TOF instrument equipped with a coiled ribbon collision surface (Waters, Wilmslow, UK).

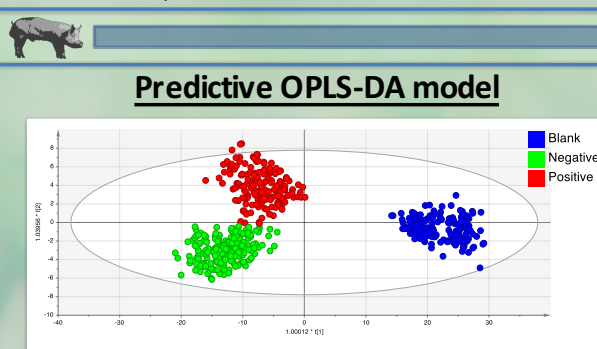

$$
\begin{array}{|l|r|r|r|r|r|}
\hline & \text { Members } & \text { Correct } & \text { Blank } & \text { Negative } & \multicolumn{1}{c|}{\text { Positive }} \\
\hline \text { Blank } & 170 & 100 \% & 170 & 0 & 0 \\
\hline \text { Negative } & 192 & 100 \% & 0 & 192 & 0 \\
\hline \text { Positive } & 174 & 97.7 \% & 0 & 4 & 170 \\
\hline \text { Noclass } & 0 & & 0 & 0 & 0 \\
\hline \text { Total } & 536 & 99.25 \% & 170 & 196 & 170 \\
\hline
\end{array}
$$

Evaluation of the obtained OPLS-DA model showed good validation characteristics $R^{2}(Y)=0.872, Q^{2}(Y)=$ $\mathbf{0 . 7 5 6}$, indicating a good fit and predictive properties of the model. CV-ANOVA analysis ( $<<0.001$ ) and permutation testing (20 permutations) confirmed the reliability of the obtained OPLS-DA model. A total classification accuracy of $99 \%$ was achieved.

\section{Untargeted profiling \& Chemometric data processing}

Boar and sow neck fat samples were profiled, providing a mass spectral fingerprint. Afterwards, the mass spectral fingerprints were used to a construct predictive OPLS-DA model for classification. To this end, Progenesis QI and SIMCA 2.2 software were used for data pre-processing purposes and model building, respectively.

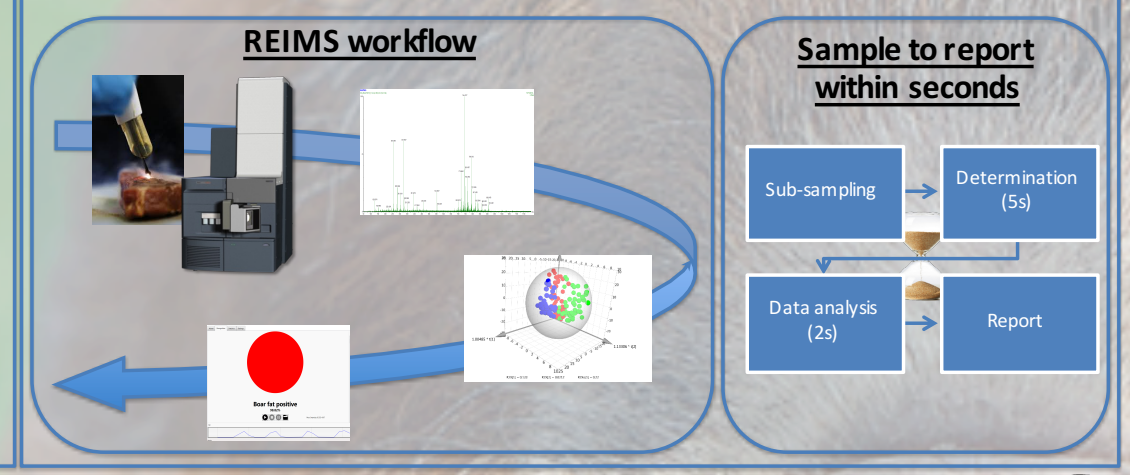

RESULTS Candidate discriminating compounds

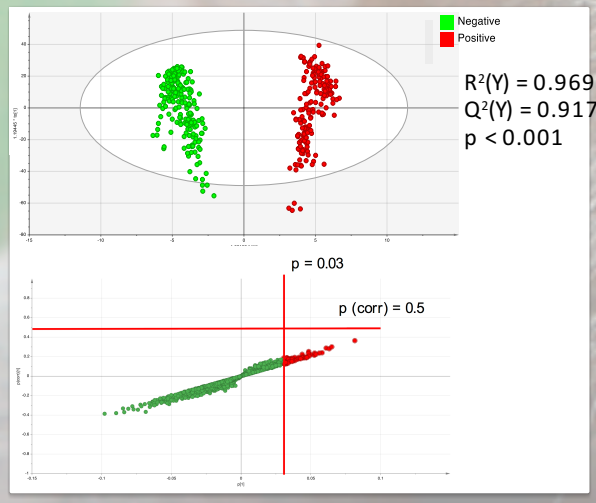

In total, 60 ions demonstrated a high contribution to the presence of boar taint in neck fat $|\mathbf{p}| \geq$ $\mathbf{0 . 0 3}$. However, none of the latter compounds were reliable $\mid \mathbf{p}$ (corr) $\mid \geq \mathbf{0 . 5}$ for allocation of the samples in the boar taint negative or positive group. Consequently, in order to correctly classify between tainted and untainted boar carcasses, the complete mass spectrum should be taken into account.

\section{CONCLUSIONS}

\section{$r x$}

In this study REIMS was able to correctly (99\% accuracy) identify tainted boar neck fat samples within a couple of seconds, based on an untarg et ed profiling approach. The discrimination between boars (tainted \& untainted) and sows originated from al terations in lipid profiles, mainly situated in the fatty acid and phospholipid region. Moreover, as REIMS en ables in-situ analysis, guaranteeing point-of-control monitoring, it is a very promising and powerful tool for a diverse range of applications in food safety and quality.

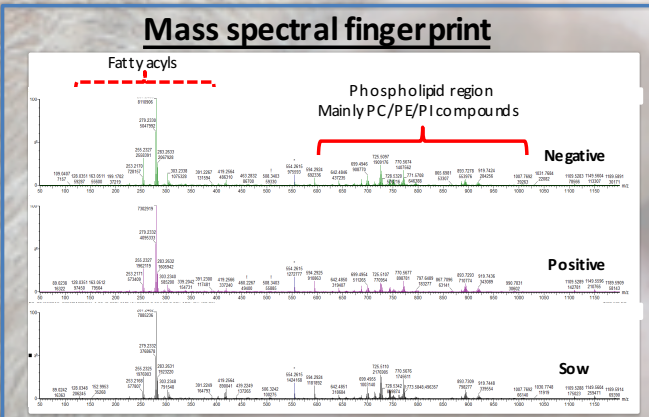

Spectral differences between the three groups were mainly situated in the phospholipid and fatty acid region. MUFAs were predominantly present in the boar taint positive group. PUFAs and SFAs were most abundant in the 2 boar groups in comparison to sows.
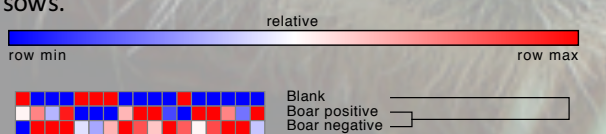

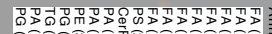

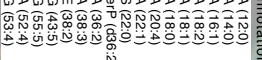

\section{ACKNOWLEDGMENTS}

Kaat Verplanken is supported by Flanders Innovation \& Entrepreneurship (VLAIO) (IWT: SB 131420).

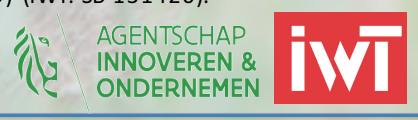

